\title{
MODULAR FORMS AND ELLIPTIC CURVES OVER THE CUBIC FIELD OF DISCRIMINANT -23
}

\author{
PAUL E. GUNNELLS AND DAN YASAKI
}

\begin{abstract}
Let $F$ be the cubic field of discriminant -23 and let $\mathcal{O} \subset F$ be its ring of integers. By explicitly computing cohomology of congruence subgroups of $\mathrm{GL}_{2}(\mathcal{O})$, we computationally investigate modularity of elliptic curves over $F$.
\end{abstract}

\section{INTRODUCTION}

1.1. Let $F$ be a number field with ring of integers $\mathcal{O}$, and let $E$ be an elliptic curve defined over $F$. According to a general contemporary philosophy with roots in the work of Taniyama, Shimura, and Weil, the curve $E$ should be modular. There are many different perspectives about what this might mean, but at the very least one expects there exists a cuspidal automorphic form $f$ on $\mathrm{GL}_{2}$ that is a Hecke eigenform with integral Hecke eigenvalues, and such that these eigenvalues are closely related to the number of points on $E$ over various finite fields. In particular, suppose $\mathfrak{n}$ is the conductor of $E$. Then one expects $f$ also to have conductor $\mathfrak{n}$, and that there should be an identification of partial $L$-functions $L^{S}(s, f)=L^{S}(s, E)$, where $S$ is a fixed finite set of primes including all those dividing $\mathfrak{n}$. This identification is done through matching of Euler factors at good primes: if $\mathfrak{p}$ is prime to $\mathfrak{n}$, then one wants

$$
\left|E\left(\mathbb{F}_{\mathfrak{p}}\right)\right|=\operatorname{Norm}(\mathfrak{p})+1-a_{\mathfrak{p}},
$$

where $\mathbb{F}_{\mathfrak{p}}=\mathcal{O} / \mathfrak{p}$ is the residue field and $a_{\mathfrak{p}} \in \mathbb{Z}$ is the $\mathfrak{p t h}$ Hecke eigenvalue. This idea has been computationally investigated by a variety of authors, including Cremona and his students for $F$ complex quadratic [7, 9, 10, 21]; Socrates-Whitehouse and Dembélé for $F$ real quadratic [11,23] ; and the current authors, in joint work with F. Hajir, for $F$ the $C M$ field of fifth roots of unity [15].

In this paper, we continue this computational work and study the modularity of elliptic curves when $F$ is the complex cubic field of discriminant -23 . This field has signature $(1,1)$ and is thus not Galois (its Galois closure is the Hilbert class field of $\mathbb{Q}(\sqrt{-23})$ ).

The overall program is similar to that of [15]. Namely, instead of explicitly working with automorphic forms, we work with the cohomology of the congruence subgroups $\Gamma_{0}(\mathfrak{n}) \subset \mathrm{GL}_{2}(\mathcal{O})$. By Franke's proof of Borel's conjecture [13, one knows that this cohomology is built from certain automorphic forms, and that there is a

Date: June 19, 2018.

1991 Mathematics Subject Classification. Primary 11F75; Secondary 11F67, 11G05, 11Y99.

Key words and phrases. Automorphic forms, cohomology of arithmetic groups, Hecke operators, elliptic curves.

PG was partially supported by NSF grant DMS 1101640. We thank Avner Ash, Farshid Hajir, Alan Reid, and Siman Wong for helpful conversations. We also thank the referee for many useful suggestions. 
subspace of cuspidal cohomology that corresponds to certain cuspidal automorphic forms. This cohomology can be computed using topological tools; in particular the first step is carrying out a version of explicit reduction theory due to Koecher [20. This reduction theory is one generalization of Voronoi's theory of perfect quadratic forms [24] to a much broader setting that includes quadratic forms over both number fields of arbitrary signature and the quaternions. To the best of our knowledge, this is the first time Koecher's work has been used for such computations. Since we expect that these techniques will be useful for later researchers, we take some time to explain Koecher's work.

The second step is computing the cohomology and its decomposition under the action of the Hecke operators. Computing the cohomology is straightforward, since the reduction theory provides us with an explicit cell complex with an action by $\Gamma_{0}(\mathfrak{n})$. Computing the Hecke operators, however, is much more involved. Our technique is to use the sharbly complex and a variant of an algorithm described by the first-named author for subgroups of $\mathrm{SL}_{4}(\mathbb{Z})[17$, and later developed by both of us in 15, 18, for subgroups of $\mathrm{GL}_{2}(\mathcal{O})$, where $F$ is real quadratic and totally complex quartic. Although these settings seem to have little to do with each other, they share the feature that the highest cohomological degree where the cusp forms can contribute is exactly one less than the virtual cohomological dimension. Thus although the symmetric spaces and underlying fields are completely different, computing the Hecke operators reduces to a similar combinatorial problem. The technique we use is closely related to that of modular symbols [4, 22, although the combinatorics are more complicated since we need to work with a different cohomology group. As in the papers [15, 17, 18, we do not have a proof that our technique of computing Hecke operators works, but the technique has always been successful in practice.

With the first two steps completed, we find ourselves with a list of Hecke eigenclasses, some of which apparently correspond to cuspforms, and further some of which have eigenvalues that are rational integers. The final step is to compile a list of elliptic curves over $F$ of small conductors. This is done in the obvious way: we enumerate curves by searching over a box of Weierstrass equations and throwing out duplicates.

After all three steps are done, we arrive at a list of cohomology classes and a list of elliptic curves. We found complete agreement between these lists. In particular:

- For each elliptic curve $E$ with norm conductor within the range of our cohomology computations, we found a cuspidal cohomology class with rational Hecke eigenvalues that matched the point counts for $E$ as in (1), for every Hecke operator that we checked.

- For each cuspidal cohomology class with rational Hecke eigenvalues, we found a corresponding elliptic curve whose point counts matched every eigenvalue we computed.

We now give a guide to the contents of the paper. In $\S ₫ 23$ we recall Koecher's work on positivity domains, and explain how one can find explicit fundamental domains for $\mathrm{GL}_{n}$ over number fields acting on spaces of quadratic forms. In $\$ 4$ we compute the Koecher polyhedron for the case under consideration, namely $\mathrm{GL}_{2}(\mathcal{O})$ where $\mathcal{O}$ is the ring of integers in the cubic field of discriminant -23 . Next, \$5 explains the complexes we use to compute the cohomology of subgroups of $\mathrm{GL}_{2}(\mathcal{O})$ and the Hecke action. After this, in $₫ 6$ we give a brief overview of how we compute 
the action of the Hecke operators, following [15, 18. The following two sections $\S \$ 7 / 8$ give more details about what is done differently in the current paper from the techniques in [15, 18. Finally, in $\$ 9$ we present our computational data.

\section{Positivity domains}

In this section we review Koecher's study of positivity domains [20, which generalizes Voronoi's reduction theory for positive definite quadratic forms 24.

Let $V$ be a finite dimensional vector space over $\mathbb{R}$. Let $\langle\rangle:, V \times V \rightarrow \mathbb{R}$ be a positive definite symmetric bilinear form. The form determines a norm $|\cdot|$ on $V$ in the usual way by $|v|=\sqrt{\langle v, v\rangle}$, and we give $V$ the metric topology. For any subset $C \subset V$, let $\bar{C}$ be the closure, let $\operatorname{Int}(C)$ be the relative interior, and let $\partial C=\bar{C} \backslash \operatorname{Int}(C)$ be the boundary.

Definition 2.1. A subset $C \subset V$ is called a positivity domain if the following hold:

- $C$ is open and nonempty.

- $\langle x, y\rangle>0$ for all $x, y \in C$.

- For each $x \in V \backslash C$ there is a nonzero $y \in \bar{C}$ such that $\langle x, y\rangle \leq 0$.

One can show that a positivity domain $C$ is a convex cone in $V$. In other words, if $y \in C$ then $\lambda y \in C$ for all $\lambda>0$, and if $y, y^{\prime} \in C$ then $y+y^{\prime} \in C$. Furthermore, $C$ contains no line.

Let $D \subset \bar{C} \backslash\{0\}$ be a nonempty discrete subset. For $y \in C$, let

$$
\mu(y)=\operatorname{Inf}_{d \in D}\{\langle d, y\rangle\} .
$$

Koecher proves that $\mu(y)>0$ and that the infimum is achieved only on a finite set of points, which we denote $M(y)$ :

$$
M(y)=\{d \in D \mid\langle d, y\rangle=\mu(y)\} .
$$

We call $M(y)$ the set of minimal vectors for $y$.

Definition 2.2. A point $y \in C$ is called perfect if the linear span of its minimal vectors $M(y)$ is all of $V$.

Note that the set $M(y)$, as well as the notion of perfection of a point in $C$, depend on the choice of the set $D$. If $y$ is perfect, then so is $\lambda y$ for $\lambda>0$, and clearly $M(\lambda y)=M(y)$. We let $\Phi=\Phi(D)$ be the set of all perfect points $y$ with $\mu(y)=1$.

Example 2.3. We consider the classical case of this construction. Let $V$ be the space of symmetric $n \times n$ matrices over $\mathbb{R}$, let $\langle a, b\rangle=\operatorname{Tr}(a b)$, and let $C \subset V$ be the cone of positive definite matrices. Let $D \subset V$ be the set of points of the form $v v^{t}$ for $v \in \mathbb{Z}^{n}$, where we regard elements of $\mathbb{Z}^{n}$ as column vectors. Then if $y \in C$, $d=v v^{t} \in D$, the quantity $\langle d, y\rangle$ is easily seen to be the value $Q_{y}(v)=v^{t} y v$, in other words the value of the positive definite quadratic form $Q_{y}$ determined by $y$ on the integral vector $v$. Koecher's notion of perfect coincides with Voronoi's notion of a perfect quadratic form: a positive definite quadratic form is perfect if it can be recovered from the knowledge of its nonzero minimum and the set of vectors on which the minimum is attained.

Returning now to the general setting, Voronoi used perfect quadratic forms to provide an explicit reduction theory for the cone of positive definite quadratic forms, 
and we want the same for our positivity domain $C$. Unfortunately not all sets $D$ will accomplish this. Thus we have the following definition:

Definition 2.4. A nonempty discrete subset $D \subset \bar{C} \backslash\{0\}$ is said to be admissible if for any sequence $\left\{y_{i}\right\}$ converging to a point in $\partial C$, we have $\lim \mu\left(y_{i}\right)=0$.

Note that the set $D$ from Example 2.3 is admissible. Suppose $\left\{y_{i}\right\} \rightarrow y \in \partial C$. The real quadratic form $Q_{y}$ associated to $y$ is degenerate, and thus there is a nonzero subspace $W \subset \mathbb{R}^{n}$ such that $Q_{y}$ restricted to $W$ vanishes. Since there exist integral vectors arbitrarily close to $W$, we have $\lim \mu\left(y_{i}\right)=0$.

Koecher proved that if $D$ is admissible, then $\Phi(D)$ is a discrete subset of $C$, and provides a polyhedral decomposition of $C$. To explain what we mean, we must recall some notions from convex geometry (a convenient reference is 14, Chapter 1]). Recall that a polyhedral cone in a real vector space $V$ is a subset $\sigma$ of the form

$$
\sigma=\sigma\left(v_{1}, \ldots, v_{p}\right)=\left\{\sum_{i=1}^{p} \lambda_{i} v_{i} \mid \lambda_{i} \geq 0\right\},
$$

where $v_{1}, \ldots, v_{p}$ is a fixed set of vectors. We say that the $v_{1}, \ldots, v_{p}$ span $\sigma$. The dimension of $\sigma$ is the dimension of its linear span; if the dimension of $\sigma$ is $n$, then we call $\sigma$ an $n$-cone. If $\sigma$ is spanned by a linearly independent subset, then $\sigma$ is called simplicial.

Now given any $y \in \Phi(D)$, let $\sigma(y)$ be the cone

$$
\sigma(y)=\left\{\sum \lambda_{d} d \mid \lambda_{d} \geq 0, d \in M(y)\right\} .
$$

Koecher calls $\sigma(y)$ the perfect pyramid of $y$. We remark that typically $\sigma(y)$ is not a simplicial cone.

Let $\Sigma$ be the set of perfect pyramids and all their proper faces, as $y$ ranges over all points in $\Phi(D)$. Koecher proves that for admissible $D$, the perfect pyramids have the following properties:

(i) Any compact subset of $C$ meets only finitely many perfect pyramids.

(ii) Two different perfect pyramids have no interior point in common.

(iii) Given any perfect pyramid $\sigma$, there are only finitely many perfect pyramids $\sigma^{\prime}$ such that $\sigma \cap \sigma^{\prime}$ contains a point of $C$ (which, by item (iii), must lie on the boundaries of $\sigma, \sigma^{\prime}$ ).

(iv) The intersection of any two perfect pyramids is a common face of each.

(v) Let $\sigma(y)$ be a perfect pyramid and $F$ a codimension one face of $\sigma(y)$. If $F$ meets $C$, then there is another perfect pyramid $\sigma\left(y^{\prime}\right)$ such that $\sigma(y) \cap$ $\sigma\left(y^{\prime}\right)=F$.

(vi) We have $\bigcup_{\sigma \in \Sigma} \sigma \cap C=C$.

In item ( $\sqrt{\mathbf{v}})$, if a facet $F$ of a perfect pyramid is contained in $\partial C$, we say that $F$ is a dead end. In item (iii), the pyramid $\sigma^{\prime}$ is called a neighbor of $\sigma$. Property (iv), together with the definition of $\Sigma$, implies that $\Sigma$ is a fan [14, §1.4].

Definition 2.5. We call $\Sigma$ the Koecher fan, and the cones in $\Sigma$ the Koecher cones.

Now we bring groups into the picture. Let $G \subset \mathrm{GL}(V)$ be the group of automorphisms of $C$. Let $\Gamma \subset G$ be a discrete subgroup such that $\Gamma D=D$. Koecher proves that if $D$ is admissible, then $\Gamma$ acts properly discontinuously on $C$ [20, §5.4]. Moreover, the Koecher fan gives an explicit reduction theory for $\Gamma$ in the following sense: 
(O1) There are finitely many $\Gamma$-orbits in $\Sigma$.

(O2) Every $y \in C$ is contained in a unique cone in $\Sigma$.

(O3) Given any cone $\sigma \in \Sigma$ with $\sigma \cap C \neq \emptyset$, the group $\{\gamma \in \Gamma \mid \gamma \sigma=\sigma\}$ is finite.

Choose representatives $\sigma_{1}, \ldots, \sigma_{n}$ of the orbits of $\Gamma$ in $\Sigma$, and let

$$
\Omega=\bigcup \sigma_{i} \cap C \text {. }
$$

One would hope that $\Omega$ is a fundamental domain for the action of $\Gamma$ on $C$, but unfortunately the properties $(\mathbf{O} 1)-(\mathbf{O 3})$ do not imply this. However $\Omega$ is close to a fundamental domain: each of the $\sigma_{i}$ has at worst a finite stabilizer subgroup in $\Gamma$. If one wishes to refine $\Omega$ to an exact reduction domain, as Koecher does, one must account for these finite stabilizers, for instance by passing to the barycentric subdivision of the perfect pyramids and then taking an appropriate union of the resulting cones. For our purposes this is not necessary, since explicit knowledge of $\Omega$ and these stabilizers suffices for cohomology computations (cf. [1, §4]).

We conclude this section by presenting another viewpoint on the perfect pyramids and the Koecher fan. This perspective does not appear in [20, but instead is motivated by the theory of cores and co-cores in [3, Chapter II].

Definition 2.6. Let $D$ be an admissible set in $\bar{C}$. The Koecher polyhedron $\Pi$ is the convex hull in $\bar{C}$ of $D$.

The connection between $\Pi$ and $\Sigma$ is given by the following proposition:

Proposition 2.7. Assume that no perfect pyramid in $\Sigma$ has a dead end. Then the nonzero cones in the fan $\Sigma$ are the cones on the faces of $\Pi$.

Proof. Because $\Pi$ is a convex polyhedron, it suffices to see that the perfect pyramids are the cones on the facets (maximal proper faces) of $\Pi$. So let $y$ be a perfect form with minimal vectors $d_{1}, \ldots, d_{n}$ and with perfect pyramid $\sigma(y)$. The equation $\langle x, y\rangle=1$ defines a hyperplane in $V$ that is a supporting hyperplane for $\Pi$ (all other $d \in D$ satisfy $\langle d, y\rangle>1$, and $\Pi$ is the convex hull of $D$ ). Since the $d_{i}$ span $V$, it follows that the convex hull of the $d_{i}$ is a facet of $\Pi$. Thus $\sigma(y)$ gives rise to a facet $F$ of $\Pi$, and $\sigma(y)$ is the cone on $F$. Since we assume no perfect pyramids have dead ends, property $(\mathbb{\nabla})$ on page 4 implies that the facets of $\Pi$ meeting $F$ in a codimension one face correspond to perfect forms by a similar argument. Thus the facets of $\Pi$ are in bijection with the perfect pyramids.

\section{QUADRATIC FORMS OVER NUMBER FIELDS AND REDUCTION THEORY}

In this section we specialize the results of $\$ 2$ to the setting of primary concern to us. This recapitulates [20, $\S 9]$.

Let $F$ be a number field of degree $d=r+2 s$ with $r$ real embeddings and $s$ conjugate pairs of complex embeddings. For each pair of complex conjugate embeddings, choose and fix one. We can then identify the infinite places of $F$ with its real embeddings and our choice of complex embeddings.

For each infinite place $v$ of $F$, let $V_{v}$ be the real vector space of $n \times n$ real symmetric $\left(\right.$ respectively, of complex Hermitian) matrices $\operatorname{Sym}_{n}(\mathbb{R})\left(\operatorname{resp} ., \operatorname{Herm}_{n}(\mathbb{C})\right)$ if $v$ is real (resp., complex). Let $C_{v}$ be the corresponding cone of positive definite (resp., positive Hermitian) forms. Put $V=\prod_{v} V_{v}$ and $C=\prod_{v} C_{v}$, where the products 
are taken over the infinite places of $F$. We equip $V$ with the inner product

$$
\langle x, y\rangle=\sum_{v} c_{v} \operatorname{Tr}\left(x_{v} y_{v}\right)
$$

where the sum is again taken over the infinite places of $F$, and $c_{v}$ equals 1 if $v$ is real and equals 2 if $v$ is complex.

The group $G=\mathrm{GL}_{n}(\mathbb{R})^{r} \times \mathrm{GL}_{n}(\mathbb{C})^{s}$ acts on $V$ by

$$
(g \cdot y)_{v}= \begin{cases}g_{v} y_{v} g_{v}^{t} & v \text { real } \\ g_{v} y_{v} \bar{g}_{v}^{t} & v \text { complex. }\end{cases}
$$

This action preserves $C$, and exhibits $G$ as the full automorphism group of $C$. Moreover, if $\mathbf{G}$ is the reductive group $\operatorname{Res}_{F / \mathbb{Q}} \mathrm{GL}_{n}$, then we can identify the group of real points $\mathbf{G}(\mathbb{R})$ with $G$. In fact, we can identify the quotient $C / \mathbb{R}_{>0}$ of $C$ by homotheties with the symmetric space $X=G / K A_{G}$, where $K \subset G$ is the maximal compact subgroup and $A_{G}$ is the split component (the connected component of a maximal $\mathbb{Q}$-split torus in the center of $\mathbf{G}$ ). Indeed, let $X_{n}$ (respectively $Y_{n}$ ) be the symmetric space of noncompact type $\mathrm{SL}_{n}(\mathbb{R}) / \mathrm{SO}(n)\left(\operatorname{resp} . \mathrm{SL}_{n}(\mathbb{C}) / \mathrm{SU}(n)\right)$. Then we have

$$
X \simeq\left(X_{n}\right)^{r} \times\left(Y_{n}\right)^{s} \times Z,
$$

where $Z$ is a euclidean symmetric space of dimension $r+s-1]$ Now we also have isomorphisms

$$
\operatorname{Sym}_{n}(\mathbb{R}) / \mathbb{R}_{>0} \stackrel{\sim}{\rightarrow} X_{n}, \quad \operatorname{Herm}_{n}(\mathbb{C}) / \mathbb{R}_{>0} \stackrel{\sim}{\rightarrow} Y_{n} .
$$

The identification $C / \mathbb{R}_{>0} \simeq X$ then follows, once one rewrites (3) as $\operatorname{Sym}_{n}(\mathbb{R}) \simeq$ $X_{n} \times \mathbb{R}_{>0}$ and $\operatorname{Herm}_{n}(\mathbb{C}) \simeq Y_{n} \times \mathbb{R}_{>0}$.

Now we construct an admissible subset $D$ of $\bar{C}$. Let $\mathcal{O}$ be the ring of integers of $F$. The nonzero (column) vectors $\mathcal{O}^{n} \backslash\{0\}$ determine points in $V$ via

$$
q: x \longmapsto\left(x_{v} x_{v}^{*}\right) .
$$

Here $*$ denotes transpose if $v$ is real, and conjugate transpose if $v$ is complex. We have $q(x) \in \bar{C}$ for all $x \in \mathcal{O}^{n}$, and by analogy with Voronoi's construction it is natural to consider the set $D$ obtained by applying $q$ to the nonzero points. Indeed, we have the following basic result of Koecher (cf. [20, Lemma 11]):

Proposition 3.1. The set

$$
D=\left\{q(x) \mid x \in \mathcal{O}^{n} \backslash\{0\}\right\}
$$

is admissible.

The group $\Gamma=\mathrm{GL}_{n}(\mathcal{O})$ acts on $C$ and takes $D$ into itself. Thus we can find the Koecher fan $\Sigma$. After passing to the symmetric space $X$, we obtain a decomposition of $X$ into cells with $\Gamma$-action that can then be used to compute the cohomology of $\Gamma$ and its finite-index subgroups as in [1,15] and other places.

Remark. One can think of the cone $C$ as being the space of real-valued positive quadratic forms over $F$ in $n$-variables. Specifically, if $A \in C$ is a tuple $\left(A_{v}\right)$, then $A$ determines a quadratic form $Q_{A}$ on $F^{n}$ by

$$
Q_{A}(x)=\sum c_{v} x_{v}^{*} A_{v} x_{v},
$$

\footnotetext{
${ }^{1}$ In terms of the associated discrete groups, this flat factor $Z$ accounts for the difference between $\mathrm{SL}_{n}(\mathcal{O})$ and $\mathrm{GL}_{n}(\mathcal{O})$; the former is infinite index in the latter.
} 
where $c_{v}$ is defined in (2) and $*$ is defined as in (44). However, not every quadratic form of interest comes from a matrix $A$ that is the image of a matrix from $F$ under the embeddings. For instance, in general the perfect forms will not come from matrices over $F$ in this way.

\section{The Koecher Polyhedron And FAn for the FIELD OF DISCRIMINANT -23}

From now on, we set $n=2$ and consider a particular mixed signature cubic field, namely $F=\mathbb{Q}[x] /(f(x))$, where $f(x)=x^{3}-x^{2}+1$. If we choose a root $t$ of $f$, then the field $F$ has ring of integers $\mathcal{O}=\mathbb{Z}[t]$, discriminant -23 , and class number one. We remark that much of what we do can be extended to other mixed signature cubic fields, but for this investigation we focus on $F$ to honor its special place in the menagerie of complex cubics 2

Since $F$ has signature $(1,1)$, the space of forms $V$ is the product

$$
V=\operatorname{Sym}_{2}(\mathbb{R}) \times \operatorname{Herm}_{2}(\mathbb{C})
$$

The quotient $X=C / \mathbb{R}_{>0}$ is 6 -dimensional.

We now present representatives for the $\Gamma=\mathrm{GL}_{2}(\mathcal{O})$ orbits of perfect forms. These were computed using the algorithm in [16, which is a recasting of Voronoi's original algorithm to the setting of self-adjoint homogeneous cones. In particular, the algorithm begins with an initial perfect form and then identifies the neighbors of the corresponding perfect pyramid. To find the initial perfect form, we computed a large enough part of the Koecher polyhedron $\Pi$ (Definition 2.6) to identify a facet.

In our computation, we found that there are nine $\Gamma$-orbits of perfect forms, which thus give rise to nine $\Gamma$-orbits of perfect pyramids. Seven of these orbits consist of simplicial cones (each with seven spanning vectors); the remaining two classes of cones are spanned by eight and nine vectors.

Theorem 4.1. A perfect binary form over $F$ has minimal vectors that are $\mathrm{GL}_{2}(\mathcal{O})$ conjugate to exactly one of the following sets (each vector $d$ in these lists is a

\footnotetext{
${ }^{2}$ For instance, it appears first in lists of cubic fields ordered by the absolute value of their discriminants. It appears often in algebraic number theory courses since its Galois closure is the Hilbert class field of $\mathbb{Q}(\sqrt{-23})$.
} 
representative of a pair $\pm d$ of minimal vectors):

$$
\begin{aligned}
& \left\{\left[\begin{array}{l}
1 \\
0
\end{array}\right],\left[\begin{array}{l}
0 \\
1
\end{array}\right],\left[\begin{array}{l}
1 \\
1
\end{array}\right],\left[\begin{array}{c}
t^{2}-t \\
t^{2}
\end{array}\right],\left[\begin{array}{l}
-t \\
-t
\end{array}\right],\left[\begin{array}{c}
1 \\
-t^{2}+1
\end{array}\right],\left[\begin{array}{c}
0 \\
-t
\end{array}\right]\right\}, \\
& \left\{\left[\begin{array}{l}
1 \\
0
\end{array}\right],\left[\begin{array}{l}
0 \\
1
\end{array}\right],\left[\begin{array}{l}
-t \\
-t
\end{array}\right],\left[\begin{array}{c}
1 \\
-t^{2}+1
\end{array}\right],\left[\begin{array}{c}
t^{2}-t \\
0
\end{array}\right],\left[\begin{array}{c}
0 \\
-t
\end{array}\right],\left[\begin{array}{c}
t^{2}-t \\
t^{2}
\end{array}\right]\right\} \\
& \left\{\left[\begin{array}{l}
1 \\
0
\end{array}\right],\left[\begin{array}{l}
0 \\
1
\end{array}\right],\left[\begin{array}{l}
1 \\
1
\end{array}\right],\left[\begin{array}{c}
t^{2} \\
1
\end{array}\right],\left[\begin{array}{c}
-t^{2}+1 \\
-t^{2}
\end{array}\right],\left[\begin{array}{c}
-t \\
0
\end{array}\right],\left[\begin{array}{l}
-t \\
-t
\end{array}\right],\left[\begin{array}{c}
1 \\
-t^{2}+1
\end{array}\right],\left[\begin{array}{c}
0 \\
-t
\end{array}\right]\right\}, \\
& \left\{\left[\begin{array}{l}
1 \\
0
\end{array}\right],\left[\begin{array}{l}
0 \\
1
\end{array}\right],\left[\begin{array}{l}
1 \\
1
\end{array}\right],\left[\begin{array}{c}
t^{2} \\
1
\end{array}\right],\left[\begin{array}{c}
t^{2}-t \\
t^{2}
\end{array}\right],\left[\begin{array}{c}
1 \\
-t^{2}+1
\end{array}\right],\left[\begin{array}{c}
0 \\
-t
\end{array}\right]\right\}, \\
& \left\{\left[\begin{array}{l}
1 \\
0
\end{array}\right],\left[\begin{array}{l}
0 \\
1
\end{array}\right],\left[\begin{array}{l}
1 \\
1
\end{array}\right],\left[\begin{array}{l}
-t \\
-t
\end{array}\right],\left[\begin{array}{c}
t^{2}-t \\
0
\end{array}\right],\left[\begin{array}{c}
t^{2}-t \\
t^{2}
\end{array}\right],\left[\begin{array}{c}
0 \\
-t
\end{array}\right]\right\}, \\
& \left\{\left[\begin{array}{l}
1 \\
0
\end{array}\right],\left[\begin{array}{l}
0 \\
1
\end{array}\right],\left[\begin{array}{c}
0 \\
-t
\end{array}\right],\left[\begin{array}{l}
t^{2} \\
t^{2}
\end{array}\right],\left[\begin{array}{l}
-t \\
-t
\end{array}\right],\left[\begin{array}{c}
t^{2}-t \\
0
\end{array}\right],\left[\begin{array}{c}
-1 \\
-t
\end{array}\right],\left[\begin{array}{c}
t \\
t^{2}
\end{array}\right]\right\} \\
& \left\{\left[\begin{array}{l}
1 \\
0
\end{array}\right],\left[\begin{array}{l}
0 \\
1
\end{array}\right],\left[\begin{array}{l}
1 \\
1
\end{array}\right],\left[\begin{array}{c}
t^{2}-t \\
t^{2}
\end{array}\right],\left[\begin{array}{l}
-t \\
-t
\end{array}\right],\left[\begin{array}{c}
t^{2} \\
t
\end{array}\right],\left[\begin{array}{c}
1 \\
-t^{2}+1
\end{array}\right]\right\} \\
& \left\{\left[\begin{array}{l}
1 \\
0
\end{array}\right],\left[\begin{array}{l}
0 \\
1
\end{array}\right],\left[\begin{array}{c}
t^{2} \\
t^{2}-t
\end{array}\right],\left[\begin{array}{c}
1 \\
t^{2}-t
\end{array}\right],\left[\begin{array}{c}
-t \\
0
\end{array}\right],\left[\begin{array}{c}
1 \\
t^{2}
\end{array}\right],\left[\begin{array}{c}
t^{2}-t \\
-t
\end{array}\right]\right\}, \\
& \left\{\left[\begin{array}{l}
1 \\
0
\end{array}\right],\left[\begin{array}{l}
0 \\
1
\end{array}\right],\left[\begin{array}{l}
1 \\
1
\end{array}\right],\left[\begin{array}{c}
-t \\
0
\end{array}\right],\left[\begin{array}{c}
t^{2}-t \\
-t
\end{array}\right],\left[\begin{array}{c}
-t^{2} \\
-t^{2}+t
\end{array}\right],\left[\begin{array}{l}
t^{2}-t \\
t^{2}-t
\end{array}\right]\right\} \text {. }
\end{aligned}
$$

Using the data in Theorem 4.1, we can compute the complete combinatorial structure of the fan $\Sigma$. In particular we can enumerate the $\mathrm{GL}_{2}(\mathcal{O})$-orbits of the lower-dimensional cones of $\Sigma$. The results are given in Table 3 For later purposes, we record the following facts about $\Sigma$, which represents the information we need for our purposes. Propositions 4.2 and 4.3 are proved by direct computation.

\section{Proposition 4.2.}

(i) All cones up to dimension 6 in the Koecher fan are simplicial.

(ii) All 1-cones lie in the boundary $\partial C$.

(iii) One of the two orbits of 2-cones lies in $\partial C$; a representative has spanning vectors

$$
\left\{\left[\begin{array}{l}
1 \\
0
\end{array}\right],\left[\begin{array}{l}
t \\
0
\end{array}\right]\right\}
$$

The other orbit, with representative spanned by

$$
\left\{\left[\begin{array}{l}
1 \\
0
\end{array}\right],\left[\begin{array}{l}
0 \\
1
\end{array}\right]\right\}
$$

meets $C$. 
(iv) There are ten orbits of 3-cones, represented by

$$
\begin{gathered}
\left\{\left[\begin{array}{l}
1 \\
0
\end{array}\right],\left[\begin{array}{l}
0 \\
1
\end{array}\right],\left[\begin{array}{c}
0 \\
-t
\end{array}\right]\right\},\left\{\left[\begin{array}{l}
1 \\
0
\end{array}\right],\left[\begin{array}{l}
0 \\
1
\end{array}\right],\left[\begin{array}{c}
1 \\
-t^{2}+1
\end{array}\right]\right\},\left\{\left[\begin{array}{l}
1 \\
0
\end{array}\right],\left[\begin{array}{l}
0 \\
1
\end{array}\right],\left[\begin{array}{c}
t-1 \\
-t+1
\end{array}\right]\right\}, \\
\left\{\left[\begin{array}{l}
1 \\
0
\end{array}\right],\left[\begin{array}{l}
0 \\
1
\end{array}\right],\left[\begin{array}{l}
-t \\
-t
\end{array}\right]\right\},\left\{\left[\begin{array}{l}
1 \\
0
\end{array}\right],\left[\begin{array}{l}
0 \\
1
\end{array}\right],\left[\begin{array}{l}
t \\
1
\end{array}\right]\right\},\left\{\left[\begin{array}{l}
1 \\
0
\end{array}\right],\left[\begin{array}{l}
0 \\
1
\end{array}\right],\left[\begin{array}{c}
t^{2}-t+1 \\
t-1
\end{array}\right]\right\}, \\
\left\{\left[\begin{array}{l}
1 \\
0
\end{array}\right],\left[\begin{array}{l}
0 \\
1
\end{array}\right],\left[\begin{array}{l}
-t+1 \\
-t^{2}+t
\end{array}\right]\right\},\left\{\left[\begin{array}{l}
1 \\
0
\end{array}\right],\left[\begin{array}{l}
0 \\
1
\end{array}\right],\left[\begin{array}{l}
t^{2} \\
t^{2}
\end{array}\right]\right\},\left\{\left[\begin{array}{l}
1 \\
0
\end{array}\right],\left[\begin{array}{l}
0 \\
1
\end{array}\right],\left[\begin{array}{c}
-t^{2}+t \\
t^{2}-t+1
\end{array}\right]\right\}, \\
\left\{\left[\begin{array}{l}
1 \\
0
\end{array}\right],\left[\begin{array}{l}
0 \\
1
\end{array}\right],\left[\begin{array}{l}
1 \\
1
\end{array}\right]\right\} .
\end{gathered}
$$

All 3-cones meet the interior of $C$.

(v) There are thirty-one orbits of 4-cones. Representatives have spanning vectors given by the following list (each pair in this list should be supplemented by the spanning vectors of the cone (5)).

$$
\begin{aligned}
& \left\{\left[\begin{array}{c}
1 \\
-t^{2}+1
\end{array}\right],\left[\begin{array}{c}
0 \\
-t
\end{array}\right]\right\},\left\{\left[\begin{array}{l}
-t \\
-t
\end{array}\right],\left[\begin{array}{c}
0 \\
-t
\end{array}\right]\right\},\left\{\left[\begin{array}{l}
-t+1 \\
-t^{2}+t
\end{array}\right],\left[\begin{array}{c}
t^{2}-t+1 \\
t-1
\end{array}\right]\right\}, \\
& \left\{\left[\begin{array}{l}
-t \\
-t
\end{array}\right],\left[\begin{array}{c}
1 \\
-t^{2}+1
\end{array}\right]\right\},\left\{\left[\begin{array}{l}
t^{2} \\
t^{2}
\end{array}\right],\left[\begin{array}{l}
t \\
1
\end{array}\right]\right\},\left\{\left[\begin{array}{c}
-t \\
0
\end{array}\right],\left[\begin{array}{c}
0 \\
t^{2}-t
\end{array}\right]\right\},\left\{\left[\begin{array}{c}
t^{2}-t \\
t^{2}
\end{array}\right],\left[\begin{array}{c}
1 \\
-t^{2}+1
\end{array}\right]\right\}, \\
& \left\{\left[\begin{array}{c}
0 \\
-t
\end{array}\right],\left[\begin{array}{c}
t-1 \\
t^{2}-t
\end{array}\right]\right\},\left\{\left[\begin{array}{c}
-1 \\
-t+1
\end{array}\right],\left[\begin{array}{c}
-t^{2}+t \\
t^{2}-t+1
\end{array}\right]\right\},\left\{\left[\begin{array}{c}
t^{2}-t \\
t^{2}
\end{array}\right],\left[\begin{array}{l}
-t \\
-t
\end{array}\right]\right\}, \\
& \left\{\left[\begin{array}{c}
-1 \\
t^{2}-t
\end{array}\right],\left[\begin{array}{c}
t-1 \\
t^{2}-t
\end{array}\right]\right\},\left\{\left[\begin{array}{c}
-t \\
0
\end{array}\right],\left[\begin{array}{c}
-t^{2}+t \\
t^{2}-t+1
\end{array}\right]\right\},\left\{\left[\begin{array}{c}
-t \\
0
\end{array}\right],\left[\begin{array}{c}
-1 \\
-t+1
\end{array}\right]\right\} \text {, } \\
& \left\{\left[\begin{array}{c}
-1 \\
t^{2}-t
\end{array}\right],\left[\begin{array}{c}
0 \\
-t
\end{array}\right]\right\},\left\{\left[\begin{array}{l}
1 \\
1
\end{array}\right],\left[\begin{array}{c}
0 \\
-t
\end{array}\right]\right\},\left\{\left[\begin{array}{c}
t^{2}-t \\
-t^{2}+t
\end{array}\right],\left[\begin{array}{c}
-t+1 \\
t-1
\end{array}\right]\right\},\left\{\left[\begin{array}{l}
1 \\
1
\end{array}\right],\left[\begin{array}{c}
1 \\
-t^{2}+1
\end{array}\right]\right\}, \\
& \left\{\left[\begin{array}{c}
t^{2} \\
-t^{2}+1
\end{array}\right],\left[\begin{array}{c}
t \\
-t
\end{array}\right]\right\},\left\{\left[\begin{array}{c}
-t \\
0
\end{array}\right],\left[\begin{array}{c}
-t+1 \\
t-1
\end{array}\right]\right\},\left\{\left[\begin{array}{c}
-t \\
0
\end{array}\right],\left[\begin{array}{c}
t^{2}-t \\
-t^{2}+t
\end{array}\right]\right\} \\
& \left\{\left[\begin{array}{c}
t^{2}-t \\
-1
\end{array}\right],\left[\begin{array}{c}
-t+1 \\
-t^{2}+t
\end{array}\right]\right\},\left\{\left[\begin{array}{l}
1 \\
1
\end{array}\right],\left[\begin{array}{c}
t^{2}-t \\
t^{2}
\end{array}\right]\right\},\left\{\left[\begin{array}{c}
-t \\
t^{2}
\end{array}\right],\left[\begin{array}{c}
t \\
-t
\end{array}\right]\right\},\left\{\left[\begin{array}{c}
-t \\
t^{2}
\end{array}\right],\left[\begin{array}{c}
t^{2} \\
-t^{2}+1
\end{array}\right]\right\} \\
& \left\{\left[\begin{array}{c}
-t^{2}+t \\
t^{2}-t+1
\end{array}\right],\left[\begin{array}{c}
-t+1 \\
-t^{2}+t
\end{array}\right]\right\},\left\{\left[\begin{array}{c}
-t^{2} \\
t^{2}-t
\end{array}\right],\left[\begin{array}{c}
-t^{2}+t \\
-t+1
\end{array}\right]\right\},\left\{\left[\begin{array}{c}
-t^{2}+1 \\
1
\end{array}\right],\left[\begin{array}{c}
-t \\
-t^{2}+1
\end{array}\right]\right\}, \\
& \left\{\left[\begin{array}{c}
1 \\
t^{2}
\end{array}\right],\left[\begin{array}{c}
-t^{2}+1 \\
1
\end{array}\right]\right\},\left\{\left[\begin{array}{c}
t^{2} \\
1
\end{array}\right],\left[\begin{array}{c}
t^{2}-t \\
t^{2}
\end{array}\right]\right\},\left\{\left[\begin{array}{c}
-t \\
0
\end{array}\right],\left[\begin{array}{l}
t \\
1
\end{array}\right]\right\},\left\{\left[\begin{array}{c}
t^{2}-t \\
-t^{2}+t
\end{array}\right],\left[\begin{array}{c}
t^{2} \\
-t^{2}+t
\end{array}\right]\right\} \text {. }
\end{aligned}
$$

Proposition 4.3. Let $\gamma$ and $\delta$ denote the matrices

$$
\gamma=\left[\begin{array}{ll}
0 & 1 \\
1 & 0
\end{array}\right], \quad \delta=\left[\begin{array}{cc}
0 & -1 \\
1 & 0
\end{array}\right] .
$$

(i) The 2-cone that meets $C$ has stabilizer of order 8, isomorphic to $D_{4}$ generated by $\gamma$ and $\delta$.

(ii) The stabilizers of the 3-cones are given in Table 1.

(iii) The stabilizers of the 4-cones are given in Table Q2.

Remark. A consequence of the computations in this section is that the Koecher fan has no dead ends, in the sense of item ( $\mathbb{V}$ ) on page 4 . Thus the cones in the Koecher fan are in bijection with the cones on the faces of the Koecher polyhedron, as in Proposition 2.7. We expect this is always true for $\mathrm{GL}_{n}$ over number fields 
when $D$ is constructed as in Proposition 3.1. We do not know if this is true for general admissible sets $D$, although we expect not, since dead ends do occur in other generalizations of Voronoi's theory [12, §3].

\section{Cohomology, the Koecher complex, And the sharbly COMPlex}

We now turn to the cohomology spaces $H^{*}(\Gamma ; \mathbb{C})$, where $\Gamma \subset \mathrm{GL}_{2}(\mathcal{O})$ is now a congruence subgroup. This is isomorphic to $H^{*}(\Gamma \backslash X ; \mathbb{C})$. Since $X$ is 6 -dimensional, we have $H^{i}(\Gamma ; \mathbb{C})=0$ unless $0 \leq i \leq 6$. Since $\mathbf{G} \bmod$ its radical has $\mathbb{Q}$-rank 1 , by the Borel-Serre vanishing theorem [5] we have $H^{i}(\Gamma ; \mathbb{C})=0$ if $i=6$. Thus the cohomological dimension $\nu$ of any $\Gamma$ is 5 . One can show that the cuspidal cohomology (the part of the cohomology corresponding to cuspidal automorphic forms) only occurs in degrees $2,3,4$, and general results imply that we only need to compute one of these groups. Thus we focus on $H^{4}(\Gamma ; \mathbb{C})$; as we shall see this is the easiest group for us to compute.

We now describe the techniques we use to compute cohomology and the action of the Hecke operators. Similar techniques were used in 1, 15]. For more details about the complexes we use (in the setting of $\mathrm{GL}_{n} / \mathbb{Q}$ ), see 2 .

Let $\mathcal{T}$ be the Tits building for $\mathrm{GL}_{n} / F$. Thus $\mathcal{T}$ is a simplicial complex with $k$-simplices given by the proper flags in $F^{n}$ of length $(k+1)$. By the Solomon-Tits theorem, $\mathcal{T}$ has the homotopy type of a bouquet of $(n-2)$-spheres, and in particular has reduced homology concentrated in dimension $n-2$. One can construct classes in $\tilde{H}_{n-2}(\mathcal{T})$ by taking the fundamental classes of apartments: one chooses a basis $E=\left\{v_{1}, \ldots, v_{n}\right\}$ of $F^{n}$ and considers all the possible flags that can be constructed from $E$ by taking spans of permutations of subsets. By appropriately choosing signs one obtains a class $\left\langle v_{1}, \ldots, v_{n}\right\rangle \in \tilde{H}_{n-2}(\mathcal{T})$. It is known that such classes span the homology. We have an action of $\mathbf{G}(\mathbb{Q})$, and by definition, the Steinberg module $\mathrm{St}_{n}$ is the $\mathbf{G}(\mathbb{Q})$-module $\tilde{H}_{n-2}(\mathcal{T})$.

According to the Borel-Serre duality theorem [5], for any arithmetic subgroup $\Gamma \subset \mathbf{G}(\mathbb{Q})$ we have

$$
H^{\nu-k}(\Gamma ; \mathbb{C}) \stackrel{\sim}{\longrightarrow} H_{k}\left(\Gamma ; \mathrm{St}_{n} \otimes \mathbb{C}\right) .
$$

Thus to compute the cohomology of $\Gamma$, we need to take a resolution of the Steinberg module. We work with two different complexes; each has features that help us deal with the two sides of our computational problem, namely computing $H^{*}$ as well as the action of the Hecke operators. The first, the Koecher complex, comes from the geometry of the Koecher polyhedron. It has the advantage that it is finite mod $\Gamma$, but it does not admit an action of the Hecke operators. On the other hand the second, the sharbly complex, does admit a Hecke action, but unfortunately it is not finite $\bmod \Gamma$.

Recall that $\Sigma$ is the fan of Koecher cones in the closed cone $\bar{C}$. After we mod out by homotheties, the nonzero cones in $\Sigma$ determine cells in the quotient $\bar{X}=$ $(\bar{C} \backslash\{0\}) / \mathbb{R}_{>0}$. We let $K_{*}$ be the oriented chain complex on these cells, and let $\partial K_{*}$ be the subcomplex generated by the cells contained entirely in the boundary $\partial X=\bar{X} \backslash X$. The Koecher complex $\mathcal{K}_{*}$ is the quotient complex $K_{*} / \partial K_{*}$. We can construct a map

$$
\mathcal{K}_{1} \longrightarrow \mathrm{St}_{2}
$$

as follows. Let $\left\{e_{1}, e_{2}\right\}$ be the standard basis of $F^{2}$. By (iii) of Proposition 4.2 nontrivial generators of $\mathcal{K}_{1}$ correspond to the images of the oriented 2-cones in the 
$\mathrm{GL}_{2}(\mathcal{O})$-orbit of the cone spanned by $q\left(e_{1}\right), q\left(e_{2}\right)$. Orient this cone by taking the spanning points in this order, and then map it to the class of the corresponding apartment. This leads to a diagram

$$
\mathcal{K}_{*} \longrightarrow \mathrm{St}_{2}
$$

which gives the desired resolution.

We now turn to the sharbly complex. Recall that for any $x \in \mathcal{O}^{2} \backslash\{0\}$, we have constructed a point $q(x) \in \bar{C}$ (see (4)). Write $x \sim y$ if $q(x)$ and $q(y)$ determine the same point in $\bar{X}$. Let $\mathcal{S}_{k}, k \geq 0$, be the $\Gamma$-module $A_{k} / C_{k}$, where $A_{k}$ is the set of formal $\mathbb{C}$-linear sums of symbols $\mathbf{u}=\left[x_{1}, \ldots, x_{k+2}\right]$, where each $x_{i}$ is in $\mathcal{O}^{2} \backslash\{0\}$, and $C_{k}$ is the submodule generated by

(i) $\left[x_{\sigma(1)}, \ldots, x_{\sigma(k+2)}\right]-\operatorname{sgn}(\sigma)\left[x_{1}, \ldots, x_{k+2}\right]$,

(ii) $\left[x, x_{2}, \cdots, x_{k+2}\right]-\left[y, y_{2}, \ldots, y_{k+2}\right]$ if $x \sim y$, and

(iii) $\mathbf{u}$ if $x_{1}, \cdots, x_{k+2}$ are contained in a hyperplane (we say $\mathbf{u}$ is degenerate).

We define a boundary map $\partial: \mathcal{S}_{k+1} \rightarrow \mathcal{S}_{k}$ by

$$
\partial\left[x_{1}, \cdots, x_{k+2}\right]=\sum_{i=1}^{k+2}(-1)^{i}\left[x_{1}, \cdots, \hat{x}_{i}, \ldots, x_{k+2}\right],
$$

where $\hat{x}_{i}$ means omit $x_{i}$. The resulting complex $\mathcal{S}_{*}$ is called the sharbly complex. We have a map $\mathcal{S}_{0} \rightarrow \mathrm{St}_{2}$ analogous to (6), and the sharbly complex provides a resolution of $\mathrm{St}_{2}$.

Recall that all Koecher cones of dimension $\leq 6$ are simplicial (Proposition 4.2, (i)) Thus one can identify $\mathcal{K}_{i}$ with a subgroup of $\mathcal{S}_{i}$ for $i \leq 5$. This identification is compatible with the boundary maps, so we can think of $\mathcal{K}_{*}$ as being a subcomplex of $\mathcal{S}_{*}$ in these degrees. Since our main focus is computing $H^{4}$, we will work exclusively with Koecher/sharbly chains in degrees $0,1,2$, and therefore we regard the corresponding Koecher chains as living in the sharbly complex. Propositions 4.24 .3 give all the information we need for computing $H^{4}$.

To compute the Hecke operators, one proceeds as follows. First one computes the cohomology group $H^{4}(\Gamma \backslash X)$ using the relevant part of $\mathcal{K}_{*}$. Let $\xi$ be a cycle representing a class in $H^{4}$. We write $\xi$ as a finitely supported 1-sharbly cycle $\xi=\sum n(\mathbf{u}) \mathbf{u}$, where "cycle" means that the boundary vanishes modulo the action of $\Gamma$. We can compute the Hecke operator $T$ in the sharbly complex as

$$
T(\xi)=\sum n(\mathbf{u}) \sum_{g} g \cdot \mathbf{u},
$$

where the inner sum is taken over a finite subset of $\mathbf{G}(\mathbb{Q})$. The right hand side of (8) will usually be a 1-sharbly cycle that does not come from the Koecher complex, and thus cannot obviously be written in terms of a fixed basis of $H^{4}$. Therefore one needs an algorithm to move $T(\xi)$ back to a sum of cycles coming from $\mathcal{K}_{*}$. How this is done is described in the next section.

Remark. Since $\mathcal{K}_{*}$ is not the chain complex of a simplicial complex, one cannot directly regard it as a subcomplex of $\mathcal{S}_{*}$. However, it is possible to canonically refine $\mathcal{K}_{*}$ to a complex $\tilde{\mathcal{K}}_{*}$ that does sit naturally inside the sharbly complex: one simply considers the simplicial complex on all simplices that arise by subdividing the cells in $\mathcal{K}_{*}$ without adding new vertices. One must also add new relations that encode when an original Koecher cell is a union of simplices. Since we do not need this construction in our paper, we omit the details. 


\section{Reduction Algorithm}

In this section we describe how Hecke images $T(\xi)$ as in (8) are rewritten as a sum of sharbly cycles supported on Koecher cones. The main ideas and steps already appear in [18, which studied the case of a $\mathrm{GL}_{2}(\mathcal{O})$, where $\mathcal{O}$ is the ring of integers of a real quadratic field. Here we focus on the differences between that case and the current. We begin with some definitions.

Given a point $q(x), x \in \mathcal{O}^{2} \backslash\{0\}$, let $R(x)$ be the unique point $y \in \mathcal{O}^{2} \backslash\{0\}$ such that $y \sim x$ and $y$ is closest to the origin along the ray $\mathbb{R}_{\geq 0} q(x)$. We call $R(x)$ the spanning point of $x$; for any sharbly $\mathbf{u}=\left[x_{1}, \ldots, x_{n}\right]$ we can speak of its set of spanning points. Any sharbly $\mathbf{u}=\left[x_{1}, \ldots, x_{n}\right]$ determines a closed cone $\sigma(\mathbf{u})$ in $\bar{C}$. We call a sharbly $\mathbf{u}=\left[x_{1}, \ldots, x_{n}\right]$ reduced if its spanning points are a subset of the spanning points of some fixed Koecher cone; similarly we call a sharbly cycle reduced if each sharbly in its support is reduced. Note that $\mathbf{u}$ reduced does not mean that $\sigma(\mathbf{u})$ is the face of some perfect pyramid, and thus a reduced sharbly cycle need not come from a Koecher cycle. However, it is clear that there are only finitely many reduced sharbly cycles modulo $\Gamma$. Moreover, it is not difficult to write a reduced sharbly cycle in terms of Koecher cycles directly, so our main challenge is to rewrite $T(\xi)$ in terms of reduced sharbly cycles.

We now introduce notions of size and reduction level help us gauge how close a sharbly cycle is to being reduced. For any 0 -sharbly $\mathbf{u}=\left[x_{1}, x_{2}\right]$, define the size of $\mathbf{u}$ by $\operatorname{Size}(\mathbf{u})=\left|\operatorname{Norm}\left(\operatorname{det}\left(R\left(x_{1}\right), R\left(x_{2}\right)\right)\right)\right|$. We extend this to general sharblies by first defining the size of $\mathbf{u}=\left[x_{1}, \ldots, x_{n}\right]$ to be the maximum of the size of the sub 0 -sharblies $\left[x_{i}, x_{j}\right]$, and then to chains $\xi=\sum n(\mathbf{u}) \mathbf{u}$ by taking the maximum size found over the support of $\xi$.

We now focus on 0 - and 1-sharblies. Given a 1-sharbly $\mathbf{u}=\left[x_{1}, x_{2}, x_{3}\right]$, we call its sub 0-sharblies edges, and define the reduction level of $\mathbf{u}$ to be the number of edges that are not reduced. According to Proposition 4.2 the only $\mathrm{GL}_{2}(\mathcal{O})$-class of reduced 0 -sharbly is $\left[e_{1}, e_{2}\right]$, and all we can often detect nonzero reduction level by computing sizes, but we remark that there are subtleties: for instance, there exist nonreduced 1-sharblies of reduction level zero. Similar phenomena occur in 15, 18, and reflect the infinite order units in $\mathcal{O}$.

There are also edges with size 0 that are not reduced. More precisely, looking at the edges of the standard 1-sharblies, we see that $[x, c x]$ is reduced only if the coordinates of $x$ generate the ideal $\mathcal{O}$ and $c \in\left\{ \pm t, \pm t^{-1}\right\}=\left\{ \pm \epsilon, \pm \epsilon^{-1}\right\}$, where $\epsilon=-t$ is a generator of $\mathcal{O}^{\times} \bmod$ torsion. Such 0 -sharblies are of course degenerate and are eliminated in the defining relations of $\mathcal{S}_{*}$, but when one wants to write a 1-sharbly cycle in terms of reduced cycles they must be considered. Again, this is not surprising since the same phenomena appear in [15,18]. However, if a 1-sharbly contains an edge of the $[x, \pm x]$, then the 1-sharbly is degenerate and is thrown away.

We now turn to the reduction algorithm. The overall structure proceeds as described in [18, and we refer to there for more details. For each 1-sharbly $\mathbf{u}$ in the support of a Hecke image, we $\Gamma$-equivariantly choose a collection of reducing points for the nonreduced edges of $\mathbf{u}$ (see [18, §3.3]). These points, together with the original spanning points of $\mathbf{u}$, are assembled into a new 1-sharbly chain $\xi^{\prime}$, following the cases described in [18, §3.5]. The process is repeated until the 1-sharbly chain is reduced.

In the current work, there are two new features to this algorithm: 
(i) The case of reduction level 0 is handled slightly differently than in [15, 18, 3

(ii) A new technique is used to choose reducing points $\Gamma$-equivariantly.

For item (ii) see the next section; item (iii) is discussed in 8 ,

\section{Reduction LEVEL 0}

We consider 1-sharblies with all edges reduced, but that are not themselves reduced. According to Proposition 4.2, there are two $\mathrm{GL}_{2}(\mathcal{O})$-orbits of 2-cones. One is degenerate, and the other corresponds to a 0 -sharbly of size 1 . It follows that the 1-sharblies with all edges reduced must have edges of determinant 0 or $\epsilon^{k}$, where $\epsilon$ is an infinite generator of the unit group $\mathcal{O}^{\times}$, and $k \in \mathbb{Z}$ has large absolute value. More precisely, write

$$
\mathcal{O}^{\times} \simeq\langle-1\rangle \times\langle\epsilon\rangle .
$$

Then up to $\mathrm{GL}_{2}(\mathcal{O})$-equivalence, we need only consider 1-sharblies with spanning points $e_{1}, e_{2}$, and $v=\left[\begin{array}{l}a \\ b\end{array}\right]$, where

- $a=(-1)^{f_{a}} \epsilon^{n_{a}}$ and $b=(-1)^{f_{b}} \epsilon^{n_{b}}$,

- at least one of $\left|n_{a}\right|,\left|n_{b}\right|$ is strictly greater than 1 , and

- both $n_{a}, n_{b}$ are non-zero.

We now describe how to write $\left[e_{1}, e_{2}, v\right]$ in terms of reduced 1-sharblies. We do this by replacing $\left[e_{1}, e_{2}, v\right]$ by a sum of 1 -sharblies that are $\mathrm{GL}_{2}(\mathcal{O})$-equivalent to 3 -cones listed in Proposition 4.2, First introduce new vertices $v_{a}=\left[\begin{array}{l}a \\ 0\end{array}\right], v_{b}=\left[\begin{array}{l}0 \\ b\end{array}\right]$ and edges $\left[v_{a}, v\right],\left[v_{a}, e_{1}\right],\left[v_{a}, e_{2}\right],\left[v_{b}, v_{a}\right],\left[v_{b}, v\right]$, and $\left[v_{b}, e_{2}\right]$ (cf. the left of Figure[1)

Now we treat each region $A_{i}$ separately, depending on a variety of cases. Given $z=(-1)^{f} \epsilon^{n} \in \mathcal{O}^{\times}$, let $\mathcal{I}(a) \subset \mathcal{O}^{\times}$denote the list

$$
\mathcal{I}(z)=\left[z_{0}, z_{1}, z_{2}, \ldots, z_{|n|}\right],
$$

where $z_{i}=(-1)^{f} \epsilon^{i}$ if $n \geq 0$, and $z_{i}=(-1)^{f} \epsilon^{-i}$ if $n<0$. For example, if $z=-\epsilon^{3}$, then $\mathcal{I}(z)$ is the list $\left[-1,-\epsilon,-\epsilon^{2},-\epsilon^{3}\right]$.

By construction, the region $A_{0}$ is already reduced. In fact, we have

$$
\left[v, v_{a}, v_{b}\right]=\gamma \cdot\left[e_{1}+e_{2}, e_{1}, e_{2}\right]
$$

where $\gamma=\left[\begin{array}{ll}a & 0 \\ 0 & b\end{array}\right] \in \mathrm{GL}_{2}(\mathcal{O})$.

For the regions $A_{1}, A_{2}$, along the edge $\left[v_{a}, e_{1}\right]$ we introduce vertices

$$
\varepsilon_{a}=\left\{\left[\begin{array}{l}
z \\
0
\end{array}\right] \mid z \in \mathcal{I}(a)\right\}
$$

Note that $\# \mathcal{I}(a) \geq 2$. If $\# \mathcal{I}(a)=2$, then no new vertices are added. Otherwise, let $\varepsilon_{a}^{0}$ be $\varepsilon_{a}$ with the first and last vector removed. Construct edges

$$
\begin{gathered}
\left\{[w, v] \mid w \in \varepsilon_{a}^{0}\right\}, \quad\left\{\left[w, e_{2}\right] \mid w \in \varepsilon_{a}^{0}\right\}, \quad \text { and } \\
\left\{\left[\left(\varepsilon_{a}\right)_{i},\left(\varepsilon_{a}\right)_{i+1}\right] \mid i=1, \ldots, \# \varepsilon_{a}-1\right\} .
\end{gathered}
$$

In $A_{1}$ this creates the 1 -sharblies $\left[v,\left(\varepsilon_{a}\right)_{i},\left(\varepsilon_{a}\right)_{i+1}\right]$. These are reduced. Indeed, if $n_{a} \geq 0$, then

$$
\left[v,\left(\varepsilon_{a}\right)_{i},\left(\varepsilon_{a}\right)_{i+1}\right]=\gamma \cdot\left[e_{1}, e_{2}, \epsilon e_{2}\right]
$$

\footnotetext{
${ }^{3}$ We also remark that for reduction level 1, only the second case of [18 $\S 3.5$ (III)] is needed.
} 
where $\gamma=\left[\begin{array}{cc}0 & b^{-1} \\ \epsilon^{-i} & a b^{-1} \epsilon^{-i}\end{array}\right] \in \mathrm{GL}_{2}(\mathcal{O})$. If $n_{a}<0$, then

$$
\left[v,\left(\varepsilon_{a}\right)_{i},\left(\varepsilon_{a}\right)_{i+1}\right]=\gamma \cdot\left[e_{1}, e_{2}, \epsilon^{-1} e_{2}\right],
$$

where $\gamma=\left[\begin{array}{cc}0 & b^{-1} \\ \epsilon^{i} & a b^{-1} \epsilon^{i}\end{array}\right] \in \mathrm{GL}_{2}(\mathcal{O})$. A similar argument applies to the new 1sharblies in $A_{2}$, which are $\left[e_{2},\left(\varepsilon_{a}\right)_{i+1},\left(\varepsilon_{a}\right)_{i}\right]$; thus they are also reduced.

The regions $A_{3}, A_{4}$ are handled in the same manner. Along the edge $\left[e_{2}, v_{b}\right]$ we introduce the vertices

$$
\varepsilon_{b}=\left\{\left[\begin{array}{l}
0 \\
z
\end{array}\right] \mid z \in \mathcal{I}(b)\right\} .
$$

As before $\# \mathcal{I}(b) \geq 2$, and we put $\varepsilon_{b}^{0}$ to be $\varepsilon_{b}$ with the first and last vector removed. Add edges

$$
\begin{gathered}
\left\{[w, v] \mid w \in \varepsilon_{b}^{0}\right\}, \quad\left\{\left[w, v_{a}\right] \mid w \in \varepsilon_{b}^{0}\right\}, \quad \text { and } \\
\left\{\left[\left(\varepsilon_{b}\right)_{i},\left(\varepsilon_{b}\right)_{i+1}\right] \mid i=1, \ldots, \# \varepsilon_{b}-1\right\} .
\end{gathered}
$$

The 1-sharblies added in region $A_{3}$ are $\left[v_{a},\left(\varepsilon_{b}\right)_{i},\left(\varepsilon_{b}\right)_{i+1}\right]$; those added in region $A_{4}$ are $\left[v,\left(\varepsilon_{b}\right)_{i+1},\left(\varepsilon_{b}\right)_{i}\right]$. Both types are reduced as above. The result, after completing all steps in this section, is the chain of 1-sharblies depicted in the right of Figure 1

\section{NORMAL FORMS}

An important aspect of the reduction algorithm is choosing reducing points $\Gamma$ equivariantly. This means the following. Suppose that $\mathbf{u}$ and $\mathbf{u}^{\prime}$ are two 1-sharblies that appear in a 1 -sharbly cycle $\xi \bmod \Gamma$ with coefficient 1 , and that an edge $\left[x_{1}, x_{2}\right]$ of $\mathbf{u}$ cancels an edge $\left[x_{1}^{\prime}, x_{2}^{\prime}\right]$ of $\mathbf{u}^{\prime}$ when $\partial \xi$ is taken $\bmod \Gamma$; that is, there exists some $g \in \mathrm{GL}_{2}(\mathcal{O})$ such that $g \cdot\left[x_{1}, x_{2}\right]=-\left[x_{1}^{\prime}, x_{2}^{\prime}\right]$. Suppose further that these two edges are not reduced. Then when executing the reduction algorithm we begin by choosing reducing points $w, w^{\prime}$ for them, and we require that $g \cdot w=w^{\prime}$. If this $\Gamma$-equivariance is not respected, then the output of the algorithm will not be a cycle $\bmod \Gamma$.

In [18, $\S \S 3.2-3.4]$, we presented a technique to do this. First we encode a 1sharbly cycle mod $\Gamma$ as a collection of 1 -sharblies with extra data attached to their edges, called lift matrices. These are $2 \times 2$ matrices over $\mathcal{O}$ such that if two edges are $\mathrm{GL}_{2}(\mathcal{O})$-equivalent, then their lift matrices are in the same $\mathrm{GL}_{2}(\mathcal{O})$ orbit. Then when computing a reducing point for an edge, we first compute a unique representative of the $\mathrm{GL}_{2}(\mathcal{O})$-orbit of its lift matrix. This representative is then fed into the reducing point algorithm.

Thus we must explain how to compute a normal form for any lift matrix. We use the technique of pseudo-matrices and their associated Hermite normal form [8].

Definition 8.1. A pseudo-matrix is a pair $(I, A)$, where $A=\left(a_{j, l}\right)$ is an $m \times r$ matrix with entries in $F$, and $I=\left(\mathfrak{a}_{i}\right)$ is a sequence of fractional ideals called coefficient ideals.

Definition 8.2. A pseudo-matrix $(I, A)$ is in Hermite normal form if there are $s \leq m, 1 \leq i_{1}<\cdots<i_{s}$ such that for $1 \leq l<i_{j}$, we have $a_{j, l}=0, a_{j, i_{j}}=1$, and $a_{j, l}$ is reduced modulo $\mathfrak{a}_{j} \mathfrak{a}_{l}^{-1}$, and $a_{j, l}=0$ for $j>s$. 
We specialize to our case $m=k=2$. For a matrix $A$, let $A_{i}$ denote the $i$ th row. For a matrix $A \in \operatorname{Mat}_{2}(\mathcal{O})$, let $M_{A}$ denote the $\mathcal{O}$-module generated by $\left\{A_{1}, A_{2}\right\}$. A pseudo-matrix consists of a triple $\left[I_{1}, I_{2}, A\right]$, where $I_{i} \subseteq \mathcal{O}$ are ideals, and $A \in \operatorname{Mat}_{2}(\mathcal{O})$. For a pseudo-matrix $\mathcal{A}=\left[I_{1}, I_{2}, A\right]$, let $M_{\mathcal{A}}$ denote the $\mathcal{O}$-module

$$
M_{\mathcal{A}}=\left\{a_{1} A_{1}+a_{2} A_{2} \mid a_{i} \in I_{i}\right\} .
$$

Thus if $A \in \operatorname{Mat}_{2}(\mathcal{O})$ and $\mathcal{A}=[\mathcal{O}, \mathcal{O}, A]$, then $M_{A}=M_{\mathcal{A}}$.

Given a pseudo-matrix $\mathcal{A}=\left[I_{1}, I_{2}, A\right]$, Magma's HermiteForm function returns a pair $\mathcal{H}, T$, where $\mathcal{H}$ is pseudo-matrix $\left[J_{1}, J_{2}, H\right]$ in Hermite normal form, and $T \in \mathrm{GL}_{2}(F)$ satisfies

$$
M_{\mathcal{H}}=M_{\mathcal{A}} \quad \text { and } \quad H=T A .
$$

Note that while $A$ and $H$ have entries in $\mathcal{O}$, the entries of $T$ lie in $F$ in general. Note, however, that $J_{i} \cdot T_{i} \subseteq \mathcal{O}^{2}$ for $i=1,2$. Since $F$ is class number one, we can pick generators $g_{i} \in \mathcal{O}$ such that $J_{i}=g_{i} \mathcal{O}$. Then $g_{i} T_{i} \in \mathcal{O}^{2}$, and thus $\tilde{T}=\left[\begin{array}{l}g_{1} T_{1} \\ g_{2} T_{2}\end{array}\right] \in$ $\mathrm{GL}_{2}(\mathcal{O})$. Let $\tilde{H}=\left[\begin{array}{l}g_{1} H_{1} \\ g_{2} H_{2}\end{array}\right]$. If $\mathcal{A}$ comes from a matrix $A$ so that $\mathcal{A}=[\mathcal{O}, \mathcal{O}, A]$, then we have $\tilde{H}=\tilde{T} A$.

If we wish to use the theory of Hermite normal forms to produce normal forms for matrices using the remark above, we need a section to the map

$$
\mathcal{O} \rightarrow \mathcal{O} / \mathcal{O}^{\times} \text {. }
$$

Let $a \in \mathcal{O}$ be the element for which we wish to produce a representative modulo units. First construct the ideal $\mathfrak{a}=a \mathcal{O}$. Then compute a $\mathbb{Z}$-basis for $\mathfrak{a}$, and compute the Hermite normal form (over $\mathbb{Z}$ ) of the matrix of this basis. This will produce a sequence $L \subset \mathcal{O}$ which generates $\mathfrak{a}$. In Magma, we create another ideal $\mathfrak{b}$ generated by $L$. Note that

(i) $\mathfrak{b}=\mathfrak{a}$.

(ii) The generators $\mathfrak{b}$ only depend on the ideal $\mathfrak{a}$, not the original choice of generator $a$.

Since $F$ is class number one, Magma will produce a generator $a_{0}$ for $\mathfrak{b}$, which only depends on the generators $L$. This $a_{0}$ is the representative for $a$ modulo units.

This procedure, combined with Hermite normal form for pseudo matrices and the remark above allow us to, given $A \in \operatorname{Mat}_{2}(\mathcal{O})$, output a Hermite normal form $\tilde{H}$ and transformation matrix $\tilde{T}$ such that

(i) $\tilde{H}=\tilde{T} A$.

(ii) If $A^{\prime}=g A$ for some $g \in \mathrm{GL}_{2}(\mathcal{O})$, then $\tilde{H}^{\prime}=\tilde{H}$.

This completes the computation of normal forms, but there is still one implementation detail of interest. Recall that we really only need the normal form to ensure all reducing points are chosen $\Gamma$-equivariantly. A key step in computing a reducing point is computing the cone containing the barycenter of the line through $q\left(v_{1}\right)$ and $q\left(v_{2}\right)$, where $v_{i}$ are the columns of the lift matrix (cf. [18, §3.3]). By abuse of notation, we will call this the cone containing the lift matrix.

It turns out that for a typical lift matrix $A$ arising in our computations, the cone containing $A$ is much closer to the "standard" cones (i.e., cones appearing as faces of the perfect pyramids from Theorem 4.1) than the cone containing the Hermite normal form $\tilde{H}$. This means that if we use Hermite normal form as the input to 
our code that computes reducing points, then our code will waste considerable time trying to compute the cone containing $\tilde{H}$.

We address this issue as follows by caching normal forms as we compute them. Given a matrix $A \in \operatorname{Mat}_{2}(\mathcal{O})$, we compute the pseudo matrix Hermite normal form $\mathcal{H}$. If $\mathcal{H}$ shows up on our master list, we return the associated normal form $A_{0}$. If $\mathcal{H}$ is not on the master list, we set $A_{0}=A$ to be the normal form associated to $\mathcal{H}$. In practice we go ahead and compute the reducing point for $A$ as well before adding the data to the master list.

\section{Computational Results}

We conclude by presenting our computational results, from both the cohomology and elliptic curve sides. The techniques are very similar to that of [15, so we will be brief. As before our programs were implemented in Magma [6]. We remark that, as in [15], to avoid floating-point precision problems we did not use the complex numbers $\mathbb{C}$ as coefficients for $H^{4}$, but instead computed cohomology with coefficients in the large finite field $\mathbb{F}_{12379}$. We expect that the Betti numbers we report coincide with those one would compute for the group cohomology with $\mathbb{C}$-coefficients.

As in [15], we began by computing the dimension of $H^{4}$ for a large range of level. To determine if $H_{\text {cusp }}^{4} \neq 0$, we experimentally determined the dimensions of the subspace $H_{\text {Eis }}^{4}$ spanned by Eisenstein cohomology classes [19. Such classes are closely related to Eisenstein series. In particular the eigenvalue of $T_{\mathfrak{q}}$ on these classes equals $\operatorname{Norm}(\mathfrak{q})+1$. We expect that for a given level $\mathfrak{n}$, the dimension of the Eisenstein cohomology space depends only on the factorization type of $\mathfrak{n}$. Thus initially we used some Hecke operators applied to cohomology spaces of small level norm to compute the expected Eisenstein dimension for small levels with different factorization types. The result can be found in Table 4 . After compiling this table, we were able to predict which levels had cohomology in excess of the Eisenstein subspace, and thus gave candidates for cuspidal classes.

Next we computed the Hecke operators. Our primary focus was to find eigenclasses with rational eigenvalues, and in fact this was almost always the case: for all levels except level norm $529=23^{2}$, the Hecke eigenvalues were rational. Altogether we computed the cohomology at 308 different levels, which includes all ideals with level norm $\leq 835$.

We now turn to the elliptic curve side. We compiled a list of elliptic curves over $F$ of small norm conductor simply by searching over a box of Weierstrass equations. More precisely, for a positive integer $B$, let

$$
S_{B}=\left\{c_{0}+c_{1} t+c_{2} t^{2}|| c_{i} \mid \leq B, 0 \leq i \leq 2\right\},
$$

be a boxed grid of size $(2 B+1)^{3}$ inside the lattice of algebraic integers in $F$, centered at the origin. We considered equations of the form

$$
E: y^{2}+a_{1} x y+a_{3}=x^{3}+a_{2} x^{2}+a_{4} x+a_{6}, \text { with } a_{1}, a_{2}, a_{3}, a_{4}, a_{6} \in S_{B} .
$$

and took $B=2$. For each non-zero discriminant $\leq 10000000$, we kept those of norm conductor $\leq 20000$ to arrive at a list of elliptic curves of small norm conductor. This consisted of 26445 curves lying in 1518 isomorphism classes. Of course with such a naive method we have no way of knowing whether or not we have found all isomorphism - or even isogeny - classes of curves up to some bound. Nevertheless, 
this enables us to obtain a list of curves of various conductors, which must then be reconciled with the cohomology data.

Finally we compare the two sides. In every case, we found perfect agreement:

(i) In all cases where an eigenspace was one-dimensional with rational Hecke eigenvalues, we found an elliptic curve over $F$ whose point counts over finite fields agreed with our Hecke data, at least within the range where we were able to compute both sides. There were 44 levels where this occurred; the curves are given in Table 5. Generators for the conductors of these curves are given in Table 6 .

(ii) For no level/conductor of norm $\leq 835$ did we find a curve over $F$ that was not accounted for by a Hecke eigenclass, or a Hecke eigenclass that could not be matched to a curve.

(iii) In 10 levels we encountered a two-dimensional eigenspace on which the Hecke operators acted by rational scalar matrices. These were "old" cohomology classes and can be accounted for by cohomology classes at lower levels. Table 7 records the data.

(iv) At norm level $529=23^{2}$, we found a two-dimensional cuspidal subspace where the eigenvalues live in $\mathbb{Q}(\sqrt{5})$. The eigenvalues of this cohomology class match those of the weight two newform of level 23.

\section{REFERENCES}

[1] A. Ash, P. E. Gunnells, and M. McConnell, Cohomology of congruence subgroups of $\mathrm{SL}_{4}(\mathbb{Z})$, J. Number Theory 94 (2002), no. 1, 181-212.

[2] A. Ash, P. E. Gunnells, and M. McConnell, Resolutions of the Steinberg module for $G L(n)$, J. Algebra 349 (2012), 380-390.

[3] A. Ash, D. Mumford, M. Rapoport, and Y.-S. Tai, Smooth compactifications of locally symmetric varieties, second ed., Cambridge Mathematical Library, Cambridge University Press, Cambridge, 2010, With the collaboration of Peter Scholze.

[4] A. Ash and L. Rudolph, The modular symbol and continued fractions in higher dimensions, Invent. Math. 55 (1979), no. 3, 241-250.

[5] A. Borel and J.-P. Serre, Corners and arithmetic groups, Comment. Math. Helv. 48 (1973), 436-491, Avec un appendice: Arrondissement des variétés à coins, par A. Douady et L. Hérault.

[6] W. Bosma, J. Cannon, and C. Playoust, The Magma algebra system. I. The user language, J. Symbolic Comput. 24 (1997), no. 3-4, 235-265, Computational algebra and number theory (London, 1993).

[7] J. Bygott, Modular forms and modular symbols over imaginary quadratic fields, Ph.D. thesis, Exeter, 1999.

[8] H. Cohen, Advanced topics in computational number theory, Graduate Texts in Mathematics, vol. 193, Springer-Verlag, New York, 2000.

[9] J. E. Cremona, Hyperbolic tessellations, modular symbols, and elliptic curves over complex quadratic fields, Compositio Math. 51 (1984), no. 3, 275-324.

[10] J. E. Cremona and E. Whitley, Periods of cusp forms and elliptic curves over imaginary quadratic fields, Math. Comp. 62 (1994), no. 205, 407-429.

[11] L. Dembélé, Explicit computations of Hilbert modular forms on $\mathbb{Q}(\sqrt{5})$, Experiment. Math. 14 (2005), no. 4, 457-466.

[12] M. Dutour Sikirić, A. Schürmann, and F. Vallentin, A generalization of Voronoi's reduction theory and its application, Duke Math. J. 142 (2008), no. 1, 127-164.

[13] J. Franke, Harmonic analysis in weighted $L_{2}$-spaces, Ann. Sci. École Norm. Sup. (4) 31 (1998), no. 2, 181-279.

[14] W. Fulton, Introduction to toric varieties, Annals of Mathematics Studies, vol. 131, Princeton University Press, Princeton, NJ, 1993, The William H. Roever Lectures in Geometry. 
[15] P. E. Gunnells, F. Hajir, and D. Yasaki, Modular forms and elliptic curves over the field of fifth roots of unity, Experiment. Math. (to appear), 2011.

[16] P. E. Gunnells, Modular symbols for Q-rank one groups and Voronǒ reduction, J. Number Theory 75 (1999), no. 2, 198-219.

[17] P. E. Gunnells, Computing Hecke eigenvalues below the cohomological dimension, Experiment. Math. 9 (2000), no. 3, 351-367.

[18] P. E. Gunnells and D. Yasaki, Hecke operators and Hilbert modular forms, Algorithmic number theory, Lecture Notes in Comput. Sci., vol. 5011, Springer, Berlin, 2008, pp. 387-401.

[19] G. Harder, Eisenstein cohomology of arithmetic groups. The case $\mathrm{GL}_{2}$, Invent. Math. 89 (1987), no. 1, 37-118.

[20] M. Koecher, Beiträge zu einer Reduktionstheorie in Positivitätsbereichen. I, Math. Ann. 141 (1960), 384-432.

[21] M. Lingham, Modular forms and elliptic curves over imaginary quadratic fields, Ph.D. thesis, Nottingham, 2005.

[22] J. I. Manin, Parabolic points and zeta functions of modular curves, Izv. Akad. Nauk SSSR Ser. Mat. 36 (1972), 19-66.

[23] R. G. Swan, Generators and relations for certain special linear groups, Advances in Math. 6 (1971), 1-77 (1971).

[24] G. Voronor, Sur quelques propriétés des formes quadratiques positives parfaites, J. Reine Angew. Math. 133 (1908), 97-178. 
Department of Mathematics and Statistics, University of Massachusetts, Amherst, MA 01003-9305

E-mail address: gunnells@math.umass.edu

Department of Mathematics and Statistics, University of North Carolina at GreensBoro, Greensboro, NC 27402-6170

E-mail address: d_yasaki@uncg.edu 




1.

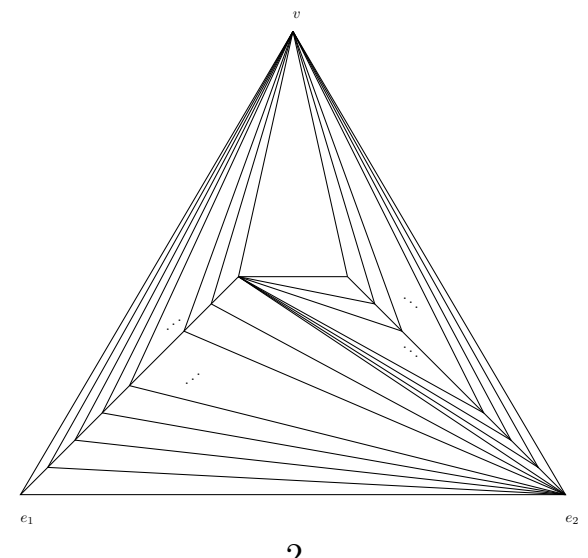

2.

Figure 1. Reduction of Type-0 1-sharbly. 


\begin{tabular}{|c|c|c|}
\hline Order & Group & Generators \\
\hline 4 & $\mathbb{Z} / 2 \mathbb{Z} \times \mathbb{Z} / 2 \mathbb{Z}$ & $-I, \gamma \delta$ \\
\hline 4 & $\mathbb{Z} / 2 \mathbb{Z} \times \mathbb{Z} / 2 \mathbb{Z}$ & $-I,\left|\begin{array}{cc}1 & -t^{2}+1\end{array}\right|$ \\
\hline 4 & $\mathbb{Z} / 2 \mathbb{Z} \times \mathbb{Z} / 2 \mathbb{Z}$ & $-I, \gamma$ \\
\hline 4 & $\mathbb{Z} / 2 \mathbb{Z} \times \mathbb{Z} / 2 \mathbb{Z}$ & $-I, \gamma$ \\
\hline 4 & $\mathbb{Z} / 2 \mathbb{Z} \times \mathbb{Z} / 2 \mathbb{Z}$ & $-I,\left|\begin{array}{cc}-1 & 0 \\
+ & 1\end{array}\right|$ \\
\hline 2 & $\mathbb{Z} / 2 \mathbb{Z}$ & $-I$ \\
\hline 2 & $\mathbb{Z} / 2 \mathbb{Z}$ & $-I$ \\
\hline 4 & $\mathbb{Z} / 2 \mathbb{Z} \times \mathbb{Z} / 2 \mathbb{Z}$ & $-I, \gamma$ \\
\hline 2 & $\mathbb{Z} / 2 \mathbb{Z}$ & $-I$ \\
\hline 12 & $D_{6}$ & $\begin{array}{cc}1 & 1 \\
-1 & 0\end{array} \mid, \gamma$ \\
\hline
\end{tabular}

TABle 1. The stabilizer groups of the 3-cones in the Koecher fan $\Sigma$. See Proposition 4.3 for notation. 


\begin{tabular}{|c|c|c|}
\hline Order & Group & Generators \\
\hline 4 & $\mathbb{Z} / 2 \mathbb{Z} \times \mathbb{Z} / 2 \mathbb{Z}$ & \begin{tabular}{l|ll}
$-I$, & 1 & $-t^{2}+1$
\end{tabular} \\
\hline 2 & $\mathbb{Z} / 2 \mathbb{Z}$ & $-I$ \\
\hline 2 & $\mathbb{Z} / 2 \mathbb{Z}$ & $-I$ \\
\hline 2 & $\mathbb{Z} / 2 \mathbb{Z}$ & $-I$ \\
\hline 2 & $\mathbb{Z} / 2 \mathbb{Z}$ & $-I$ \\
\hline 8 & $D_{4}$ & $\gamma \delta,\left[\begin{array}{cc}0 & -t^{2}+t \\
-t & 0\end{array}\right]$ \\
\hline 2 & $\mathbb{Z} / 2 \mathbb{Z}$ & $-I$ \\
\hline 2 & $\mathbb{Z} / 2 \mathbb{Z}$ & $-I$ \\
\hline 2 & $\mathbb{Z} / 2 \mathbb{Z}$ & $-I$ \\
\hline 2 & $\mathbb{Z} / 2 \mathbb{Z}$ & $-I$ \\
\hline 2 & $\mathbb{Z} / 2 \mathbb{Z}$ & $-I$ \\
\hline 2 & $\mathbb{Z} / 2 \mathbb{Z}$ & $-I$ \\
\hline 2 & $\mathbb{Z} / 2 \mathbb{Z}$ & $-I$ \\
\hline 4 & $\mathbb{Z} / 2 \mathbb{Z} \times \mathbb{Z} / 2 \mathbb{Z}$ & $-I,\left|\begin{array}{cc}-1 & t^{2}-t \\
0 & 1\end{array}\right|$ \\
\hline 4 & $\mathbb{Z} / 2 \mathbb{Z} \times \mathbb{Z} / 2 \mathbb{Z}$ & $-I,\left[\begin{array}{cc}1 & 1 \\
0 & -1\end{array}\right]$ \\
\hline 4 & $\mathbb{Z} / 2 \mathbb{Z} \times \mathbb{Z} / 2 \mathbb{Z}$ & $-I, \gamma$ \\
\hline 2 & $\mathbb{Z} / 2 \mathbb{Z}$ & $-I$ \\
\hline 2 & $\mathbb{Z} / 2 \mathbb{Z}$ & $-I$ \\
\hline 2 & $\mathbb{Z} / 2 \mathbb{Z}$ & $-I$ \\
\hline 2 & $\mathbb{Z} / 2 \mathbb{Z}$ & $-I$ \\
\hline 2 & $\mathbb{Z} / 2 \mathbb{Z}$ & $-I$ \\
\hline 2 & $\mathbb{Z} / 2 \mathbb{Z}$ & $-I$ \\
\hline 4 & $\mathbb{Z} / 2 \mathbb{Z} \times \mathbb{Z} / 2 \mathbb{Z}$ & $-I,\left[\begin{array}{cc}-t & t^{2} \\
-t & t\end{array}\right]$ \\
\hline 2 & $\mathbb{Z} / 2 \mathbb{Z}$ & $-I$ \\
\hline 2 & $\mathbb{Z} / 2 \mathbb{Z}$ & $-I$ \\
\hline 2 & $\mathbb{Z} / 2 \mathbb{Z}$ & $-I$ \\
\hline 2 & $\mathbb{Z} / 2 \mathbb{Z}$ & $-I$ \\
\hline 2 & $\mathbb{Z} / 2 \mathbb{Z}$ & $-I$ \\
\hline 4 & $\mathbb{Z} / 4 \mathbb{Z}$ & {$\left[\begin{array}{cc}t^{2} & 1 \\
-t^{2}+t & -t^{2}\end{array}\right]$} \\
\hline 4 & $\mathbb{Z} / 2 \mathbb{Z} \times \mathbb{Z} / 2 \mathbb{Z}$ & $-I,\left[\begin{array}{cc}-1 & 0 \\
t & 1\end{array}\right]$ \\
\hline 4 & $\mathbb{Z} / 2 \mathbb{Z} \times \mathbb{Z} / 2 \mathbb{Z}$ & $-I,\left[\begin{array}{cc}t^{2}-t & -t^{2}+t \\
t^{2} & -t^{2}+t\end{array}\right.$ \\
\hline
\end{tabular}

TABLE 2. The stabilizer groups of the 4-cones in the Koecher fan $\Sigma$. See Proposition 4.3 for notation. 


\begin{tabular}{|c|c|c|c|c|c|c|c|}
\hline$k$ & 1 & 2 & 3 & 4 & 5 & 6 & 7 \\
\hline$N_{k}$ & 1 & 2 & 10 & 31 & 47 & 35 & 9 \\
\hline
\end{tabular}

TABLE 3. The number $N_{k}$ of $\mathrm{GL}_{2}(\mathcal{O})$-orbits of cones of dimension $k$ in the Koecher fan $\Sigma$.

\begin{tabular}{|c|c|c|c|c|c|c|}
\hline Factorization of $\mathfrak{n}$ & $\mathfrak{p}$ & $\mathfrak{p}^{2}$ & $\mathfrak{p q}$ & $\mathfrak{p}^{3}$ & $\mathfrak{p q}^{2}$ & $\mathfrak{p q r}$ \\
\hline $\operatorname{dim} H_{\text {Eis }}^{4}\left(\Gamma_{0}(\mathfrak{n})\right)$ & 3 & 5 & 7 & 7 & 11 & 15 \\
\hline
\end{tabular}

TABLE 4. Expected dimension of Eisenstein cohomology $H_{\text {Eis }}^{4}\left(\Gamma_{0}(\mathfrak{n})\right)$ in terms of the prime factorization of $\mathfrak{n}$. Prime ideals are denoted by $\mathfrak{p}, \mathfrak{q}, \mathfrak{r}$. 


\begin{tabular}{|c|c|c|c|c|c|}
\hline $\operatorname{Norm}(\mathfrak{n})$ & $a_{1}$ & $a_{2}$ & $a_{3}$ & $a_{4}$ & $a_{6}$ \\
\hline 89 & $t-1$ & $-t^{2}-1$ & $t^{2}-t$ & $t^{2}$ & 0 \\
\hline 107 & 0 & $-t$ & $-t-1$ & $-t^{2}-t$ & 0 \\
\hline 115 & $-t^{2}+t-1$ & $-t^{2}+1$ & $t-1$ & -1 & $-t^{2}$ \\
\hline 136 & $-t^{2}$ & -1 & $-t^{2}+1$ & $t+1$ & 0 \\
\hline 161 & $t^{2}-t-1$ & $-t^{2}+t-1$ & $t^{2}-t+1$ & $t^{2}-t$ & $t-1$ \\
\hline 167 & $t^{2}+1$ & $t+1$ & $t^{2}+t-1$ & $-t^{2}-t+1$ & $-t^{2}+t+1$ \\
\hline 185 & $t$ & $-t^{2}+t+1$ & $t+1$ & 0 & 0 \\
\hline 223 & 1 & $t^{2}$ & $t^{2}+t-1$ & $-t^{2}+t-1$ & 1 \\
\hline 253 & -1 & $-t^{2}-t$ & $-t^{2}-t$ & $-t^{2}-t$ & 0 \\
\hline 259 & 0 & 1 & $-t^{2}-t-1$ & $t^{2}-t+1$ & $-t^{2}-t+1$ \\
\hline 275 & $-t^{2}+t$ & $t$ & $t^{2}-t$ & 0 & 0 \\
\hline 289 & -1 & $t^{2}-t$ & $t$ & 1 & 0 \\
\hline 293 & $t^{2}-1$ & $-t+1$ & $t^{2}-t+1$ & 0 & 0 \\
\hline 344 & $t-1$ & $-t^{2}-t$ & $-t^{2}+t+1$ & $t^{2}-1$ & 0 \\
\hline 359 & $-t^{2}+1$ & $t+1$ & $t^{2}+1$ & $t^{2}-t$ & $-t+1$ \\
\hline 385 & $-t^{2}$ & $-t^{2}-t-1$ & $-t^{2}-1$ & $t^{2}+t$ & $-t^{2}+1$ \\
\hline 392 & $-t^{2}+1$ & $-t^{2}+t+1$ & $-t+1$ & $t^{2}-1$ & $t$ \\
\hline 440 & $-t^{2}+1$ & $-t^{2}-t+1$ & $-t^{2}$ & $-t$ & 0 \\
\hline 449 & $-t^{2}$ & 1 & $-t^{2}+t+1$ & $t+1$ & 0 \\
\hline 475 & 0 & $-t$ & $t^{2}+t$ & $t^{2}-t+1$ & $-t^{2}+1$ \\
\hline 503 & $-t^{2}+t$ & $-t^{2}+t+1$ & $-t^{2}+t$ & $-t^{2}+t$ & 0 \\
\hline 505 & $t^{2}-t$ & $t^{2}-t+1$ & $t^{2}+t$ & $t^{2}-t+1$ & $-t^{2}+1$ \\
\hline 505 & $t^{2}-t-1$ & $t^{2}-1$ & 0 & $t-1$ & 0 \\
\hline 512 & 0 & $t^{2}+1$ & 0 & $t^{2}$ & 0 \\
\hline 553 & $t$ & 1 & $t$ & 0 & 0 \\
\hline 593 & $t$ & $-t-1$ & $t^{2}$ & $-t^{2}+t+1$ & 0 \\
\hline 595 & $-t^{2}+t-1$ & $-t^{2}+t+1$ & $-t^{2}+t+1$ & $-t^{2}+t+1$ & 0 \\
\hline 625 & $t$ & $-t-1$ & $t^{2}+1$ & 1 & $-t^{2}$ \\
\hline 649 & $-t^{2}-t-1$ & $-t$ & 0 & $-t^{2}+t-1$ & 0 \\
\hline 665 & $-t$ & $-t^{2}+1$ & $-t^{2}+t$ & $-t^{2}+t$ & 0 \\
\hline 685 & $t^{2}-1$ & $-t^{2}+t$ & $-t^{2}+1$ & $-t-1$ & $t^{2}$ \\
\hline 712 & $2 t^{2}-t-1$ & $-t^{2}-2 t+2$ & $t+2$ & $2 t^{2}+2 t$ & $-2 t^{2}-t$ \\
\hline 719 & $-t^{2}+t$ & $-t^{2}+t-1$ & -1 & 0 & 0 \\
\hline 719 & $t^{2}-t-1$ & $-t^{2}+t-1$ & 0 & $t^{2}-t$ & 0 \\
\hline 721 & $-t^{2}+t+1$ & $-t^{2}+t-1$ & $t^{2}+1$ & $-t-1$ & $-t+1$ \\
\hline 727 & 1 & $t^{2}+t-1$ & $t^{2}-t$ & -1 & 0 \\
\hline 773 & $2 t^{2}-1$ & $2 t+1$ & $2 t^{2}+2 t$ & $-t^{2}+2 t+1$ & $-2 t^{2}-t$ \\
\hline 805 & $-t^{2}-2 t+2$ & $-2 t^{2}+2 t$ & $t^{2}-t-1$ & $-2 t^{2}+t+2$ & $-2 t^{2}-t$ \\
\hline 808 & $-t-2$ & 0 & $2 t^{2}-t-2$ & $-2 t^{2}+2 t+2$ & $-2 t^{2}-t$ \\
\hline 809 & $-t^{2}+t-1$ & $t^{2}-1$ & $t^{2}+1$ & $t^{2}-t$ & $-t^{2}$ \\
\hline 809 & $t^{2}-1$ & $t^{2}+t-1$ & $t^{2}-t$ & $t^{2}-t$ & 0 \\
\hline 817 & $-t^{2}+t$ & $-t^{2}$ & $t^{2}-t+1$ & -1 & 0 \\
\hline 829 & 0 & $-t^{2}$ & $t^{2}-t-1$ & 0 & 0 \\
\hline
\end{tabular}

TABLE 5. Equations for elliptic curves over $F$. Here $t$ is a root of $x^{3}-x^{2}+1$. 


\begin{tabular}{|l|c|l|c|l|c|l|c|}
\hline $\mathrm{N}(\mathfrak{n})$ & generator & $\mathrm{N}(\mathfrak{n})$ & generator & $\mathrm{N}(\mathfrak{n})$ & generator & $\mathrm{N}(\mathfrak{n})$ & generator \\
\hline 89 & $4 t^{2}-t-5$ & 107 & $-5 t^{2}+3 t$ & 115 & $-2 t^{2}-2 t-3$ & 136 & $6 t^{2}-2 t-2$ \\
161 & $-5 t^{2}+5 t+4$ & 167 & $-5 t^{2}+3 t-3$ & 185 & $-t^{2}-5 t+4$ & 223 & $6 t^{2}-5 t-2$ \\
253 & $7 t^{2}-5 t-5$ & 259 & $4 t^{2}-7 t-1$ & 275 & $8 t^{2}-2 t-3$ & 289 & $3 t^{2}-7 t-2$ \\
293 & $-5 t^{2}-2 t-2$ & 344 & $6 t^{2}-2 t-8$ & 359 & $7 t^{2}-6 t-2$ & 385 & $-6 t^{2}+7 t+5$ \\
392 & $-8 t^{2}+6 t+6$ & 440 & $8 t^{2}+2 t-6$ & 449 & $t^{2}-8 t$ & 475 & $-4 t^{2}-7 t$ \\
503 & $t^{2}-t-8$ & 505 & $-2 t^{2}-7 t+2$ & 505 & $-8 t+1$ & 512 & 8 \\
553 & $9 t^{2}-4 t-2$ & 593 & $8 t^{2}-t-9$ & 595 & $11 t^{2}-4 t-6$ & 625 & $8 t^{2}+3 t+1$ \\
649 & $8 t^{2}+t-8$ & 665 & $9 t^{2}+t-8$ & 685 & $-7 t^{2}+5 t-7$ & 712 & $6 t^{2}-10 t-8$ \\
719 & $t^{2}-t-9$ & 719 & $11 t^{2}-4 t-5$ & 721 & $8 t^{2}-9$ & 727 & $10 t^{2}-7 t-7$ \\
773 & $-3 t^{2}+12 t-5$ & 805 & $3 t^{2}-6 t-10$ & 808 & $-6 t^{2}-2 t-4$ & 809 & $9 t^{2}-9 t-1$ \\
817 & $-t^{2}-7 t-8$ & 829 & $6 t^{2}-t-10$ & & & & \\
\hline
\end{tabular}

TABLE 6. Generators for ideals arising as conductors of elliptic curves. Here $t$ is a root of $x^{3}-x^{2}+1$. The two curves labelled 809 in Table 5 share the level of norm 809 in this table. The curves labelled 505 and 719 have different conductors, as indicated by the levels in this table.

\begin{tabular}{|l|c|c|}
\hline $\mathrm{N}(\mathfrak{n})$ & generator & Norm of original level \\
\hline 445 & $2 t^{2}-5 t-8$ & 89 \\
535 & $-7 t^{2}+8 t+2$ & 107 \\
575 & $-9 t^{2}+t+9$ & 115 \\
623 & $10 t^{2}+2 t-7$ & 89 \\
680 & $-6 t-8$ & 136 \\
712 & $6 t^{2}-10 t-8$ & 89 \\
749 & $-8 t^{2}+t-2$ & 107 \\
805 & $-t^{2}+10 t+4$ & 161 \\
805 & $3 t^{2}-6 t-10$ & 115 \\
835 & $-10 t^{2}+8 t-1$ & 167 \\
\hline
\end{tabular}

TABle 7. "Old" cohomology classes. In every instance the eigenspace was two-dimensional, with the Hecke operators acting by scalar matrices. The eigenvalues originally occur at the levels in the third column. Here $t$ is a root of $x^{3}-x^{2}+1$. 